\title{
First reported outbreak of locally acquired hepatitis E virus infection in Australia
}

\section{Chaturangi M Yapa $\mathrm{BSc}, \mathrm{MBChB} \mathrm{B}^{1,2}$ \\ Catriona Furlong \\ BN, MPH \\ Alexander Rosewell
PhD, MAppEpid, MIPH \\ Kate A Ward BPhty(Hons), MPH \\ Sheena Adamson \\ BVetMed, PhD \\ Craig Shadbolt \\ PhD, BSc(Hons) \\ Jen Kok \\ FRACP, FRCPA, PhD \\ Samantha L Tracy \\ Scott Bowden \\ PhD causing large outbreaks. ${ }^{1}$ HEV ge- notypes 1 and 2 predominate in these settings. ${ }^{2}$ Like other forms of acute viral hepatitis, symptoms of $\mathrm{HEV}$ include jaundice, malaise, anorexia, fever and abdominal pain. ${ }^{1}$ The incubation period is $15-64$ days. ${ }^{3}$ \\ Recently, HEV transmission has been reported in developed countries, where infection has occurred via HEV-contaminated food. Consump- tion of pork products, deer meat, wild boar and shellfish has been implicated, with HEV genotypes 3 and 4 being detected in infected per- sons. ${ }^{2,4-8}$}

Elizabeth J Smedley

BN, GDPublHealth

Mark J Ferson MD, FAFPHM, FRACP

Vicky Sheppeard MBBS, MPH(Hons), FAFPHM

Jeremy M McAnulty MBBS, MPH

I NSW Health Sydney, NSW.

2 National Centre

for Epidemiology and

Population Health

Australian National University, Canberra, ACT.

3 NSW Food Authority, Sydney, NSW.

4 Centre for Infectious Diseases, Westmead Hospital, Sydney, NSW.

5 Victorian Infectious

Diseases Reference Laboratory,

Melbourne, VIC

6 Public Health Unit,

South Eastern Sydney

Local Health District. Sydney, NSW.

7 University of

New South Wales, Sydney, NSW.

cyapa@doh.health. nsw.gov.au

doi: 10.5694/mjal5.00955

See editorial, p 254.
$\mathrm{H}$ epatitis E virus (HEV) outbreaks have not previously been reported in Australia. HEV infection mostly occurs in developing countries where transmission occurs via the faecal-oral route and contaminated water,

Pigs, in particular, may play a role in human HEV transmission. ${ }^{9}$ An increased risk of $\mathrm{HEV}$ infection associated with the consumption of processed pork products was found by a recent case-control study in the United Kingdom. ${ }^{10}$ Human and swine HEV strains exhibit a high degree of sequence homology. 5,11,12 Occupational exposure may be important, as seroprevalence rates have been found to be higher in pig veterinarians, pig farmers and abattoir workers than in healthy controls. $^{13-15}$

In Australia, HEV infection is notifiable to state and territory public health authorities. Common laboratory practice has been to test for HEV infection only in those with a history of overseas travel. Each year, 30 to 40 infections in returned travellers from HEV-endemic regions are reported, including 10 to 20 in New South Wales. $^{16}$

In October and November 2013, NSW Health was notified of two apparently unrelated cases of HEV infection within 2 weeks. Each person had been tested because of preceding

\section{Abstract}

Objective: To determine the source and extent of a locally acquired hepatitis $E$ virus (HEV) infection outbreak.

Design, setting and participants: A cluster of notified cases of HEV infection linked to a single restaurant $(X)$ was identified in May 2014. People with laboratory-confirmed HEV infection in New South Wales between January 2013 and December 2014 were interviewed about potential risk factors for HEV infection. Co-diners at restaurant $X$ and patients with suspected but unexplained viral hepatitis were retrospectively tested. Foods eaten by the infected persons were compared with those of seronegative co-diners. HEV RNA detected in sera from infected persons was sequenced and genotyped. Implicated foods were traced back to their sources.

Main outcome measures: Potential sources of infection, including overseas travel and foods eaten, and origin of implicated food products.

Results: In 55 serologically confirmed cases of HEV infection, 24 people had not travelled overseas during their incubation periods. Of the 24,17 reported having eaten at restaurant X, 15 of whom could be interviewed. All reported consuming pork liver pâté, compared with only four of seven uninfected co-diners $(P<0.05)$. The other seven people with locally acquired infections each reported consuming a pork product during their incubation periods. HEV RNA was detected in 16 of the 24 cases; all were of genotype 3. Sequencing indicated greater than 99\% homology among restaurant $X$ isolates. HEV RNA was isolated from pork sausages from a batch implicated in one of the locally acquired infections not linked with restaurant $X$. The pork livers used for pâté preparation by restaurant $X$ were traced to a single Australian farm.

Conclusions: This is the first reported HEV outbreak in Australia. HEV should be considered in patients presenting with a compatible illness, even without a history of overseas travel. Pork products should be thoroughly cooked before consumption.

overseas travel, albeit outside the incubation period for HEV infection. The HEV RNA isolated from these two people was genetically identical. A family member of one of the patients presented with symptoms of HEV infection 4 weeks later.

In May 2014, we received a further HEV notification, an infection in a man who reported that a work colleague from another state was also infected with HEV. Neither had travelled overseas during their incubation periods. The only common exposure was a meal shared with seven other colleagues at restaurant $X$, and the index patient reported that three of the seven were symptomatic. All co-diners were interviewed and tested, and HEV RNA was detected in the three symptomatic co-diners. HEV RNA from the five infected persons was genotypically identical, and also with that from two of the three 2013 cases. During routine interview of the three HEV-infected people in 2013, one had reported eating at restaurant $X$ during their incubation period, while another had not. During a follow-up interview in 2014, the third person was specifically asked about this exposure, and reported eating at restaurant $X$ during their incubation period.

In this article we report our epidemiological investigation of the source and extent of the apparent outbreak.

\section{Methods}

Epidemiological investigation

Case definition. We defined a case of $\mathrm{HEV}$ infection as a person who 
resided in NSW with laboratoryconfirmed $\mathrm{HEV}$, verified by IgG seroconversion or detection of HEVspecific IgM or HEV RNA, with an onset date (or specimen collection date, if onset date was unknown) between 1 January 2013 and 31 December 2014.

Case finding and data collected. We identified cases in three ways:

- Routine notification: As part of routine surveillance, pathology laboratories are required by the NSW Public Health Act 2010 to notify public health units of HEV infections. Surveillance specialists interview infected persons, using a standardised questionnaire. The information collected includes symptoms of illness, occupation, travel history, and water and food sources (including restaurants) during the incubation period. When an infected person had eaten at restaurant $X$, the interviewer asked about details of the food consumed there.

- Testing of co-diners from restaurant X: Co-diners of infected persons from restaurant $X$ were interviewed and tested for HEV.

- Retrospective serological surveys: We tested all sera stored at a large public laboratory, with specimen dates between 1 September 2013 and 31 May 2014, for which HEV testing had been requested but not conducted because laboratory protocols excluded testing in the absence of a relevant travel history (survey 1). We also tested sera stored at a major NSW private pathology laboratory, with specimen dates between 1 January and 31 May 2014, where the alanine transaminase (ALT) level was > 200 IU/L and hepatitis A, hepatitis B, hepatitis C, Epstein-Barr virus and cytomegalovirus infections had been excluded, but HEV testing was not performed (survey 2).

\section{Laboratory investigation}

Serology. Anti-HEV IgM and IgG were detected using HEV IgM ELISA 3.0 and HEV ELISA kits respectively (MP Diagnostics) according to the manufacturer's instructions. Reactive sera were re-tested and reported as positive if again reactive.

Viral detection and sequencing. Serum samples from confirmed cases were analysed at the Victorian Infectious Diseases Reference Laboratory. HEV RNA was extracted from serum using the QIAamp Viral RNA Mini kit (QIAGEN) and initially tested using a commercial HEV RNA polymerase chain reaction (PCR) assay (RealStar HEV RT-PCR). Samples containing HEV RNA were re-assayed by an in-house PCR assay using primers designed to amplify a portion of open reading frame (ORF) 2. The resulting PCR product was directly sequenced with internal primers. Sequences were aligned and compared with sequences in GenBank.

\section{Environmental investigation}

Investigation and food testing linked to restaurant $X$. Food handling and safety procedures at restaurant $X$ were reviewed on 15 May 2014. Preparation of pork liver pâté was observed in detail. The internal temperature of sliced pork livers was measured by inserting a thermometer into the thickest part after 3 and 4 minutes' cooking.

Three lots of chorizo sausage, three batches of cooked pork liver pâté, one sample of raw pork shoulder and raw pork jowl, one batch of cooked pork liver and eight raw pork liver samples from restaurant $X$ were collected on 15 and 22 May 2014.

After extraction and purification using the MagMax Total RNA Isolation Kit (Life Technologies), samples were tested for HEV by Advanced Analytical Australia with real-time PCR, using Hepatitis E@ceeram Tools (Ceeram).

Pork products were traced back to their source by identifying the supplier from restaurant records; through the supplier we identified the farms from which the products originated.

Testing of pork liver sausages linked with an HEV case not linked to restaurant $X$. One of the infected persons without a link to restaurant $X$ reported eating pork liver sausages during their incubation period, and had stored frozen uncooked sausages in a domestic freezer. Multiple samples were collected from several sausages and analysed for HEV at the Virology Laboratory of the Elizabeth Macarthur Agriculture Institute. Nucleic acid was purified and tested by real-time quantitative reverse transcription PCR (qRT-PCR) ${ }^{17}$ using previously published primers and probe sequences. $^{18}$

\section{Data analysis}

Responses to questionnaires administered to interviewees were transferred to a Microsoft Excel spreadsheet for analysis. Responses about food histories were analysed, and relative risks and confidence intervals calculated using Epi Info 7 (Centers for Disease Control). The Fisher exact test (two-tailed) was used to test for differences between groups; $P<0.05$ was defined as statistically significant.

\section{Ethics approval}

These studies were conducted as part of a public health investigation under the NSW Public Health Act 2010 and review by a human research ethics committee was not required.

\section{Results}

\section{Epidemiological investigation}

Notified HEV cases. Between January 2013 and December 2014, 55 cases of HEV infection were notified (Box 1). The median age of the patients was 45 years (range, 4-77 years), $36(65 \%)$ were male, and all but one $(98 \%)$ lived in metropolitan Sydney. Twenty-four (44\%) required hospitalisation, with a reported median length of stay (where known) of 7 days (range, 1-67 days). Three people (identified as co-diners of notified patients) were asymptomatic, and details about symptoms were unknown in one case. ALT levels were elevated in 33 of the 37 patients for whom they were recorded, with a median value of $1058 \mathrm{IU} / \mathrm{L}$ (range, 26-4868 IU/L; 
1 Notifications of hepatitis E virus infections in New South Wales with onset dates between January 2013 and December 2014, by likely source of acquisition*

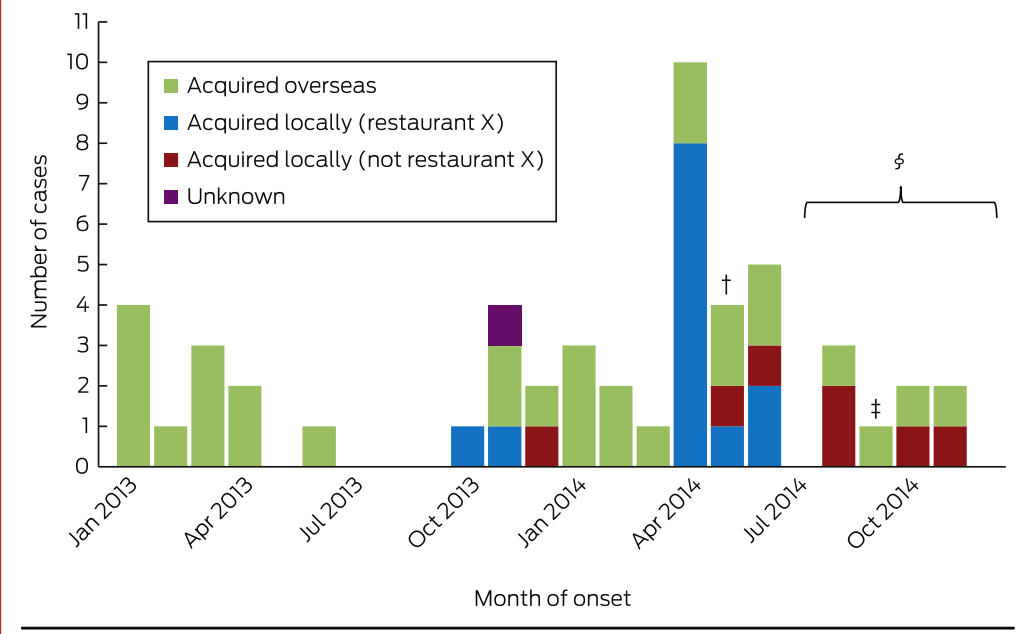

* Excludes three asymptomatic cases and one case with unknown symptom history. $\dagger$ May 2014: restaurant $X$ was inspected, and pork pâté identified as the possible source of infection; restaurant voluntarily removed pork pâté from their menu. An alert was issued to gastroenterologists and pathology laboratories. $₫$ September 2014: alert issued to general practitioners and the general public. $\S$ July-December 2014: increased HEV testing reported by the main public pathology laboratory. reference interval, 10-40 IU/L). None were pregnant.

Of the 55 patients, $30(55 \%)$ reported a history of overseas travel during their incubation periods: to South Asia (17), East Asia (six), South-East Asia (two), Africa (two), Europe (two), or the Middle East (one). One patient could not be contacted; the remaining $24(44 \%)$ did not report overseas travel.

Restaurant X outbreak. Restaurant $X$ mainly served dishes suitable for sharing by a group. The menu included more than 28 meat, seafood and vegetarian options. Seventeen

2 Characteristics of infected diners and healthy co-diners at restaurant X, October 2013 - May 2014

\begin{tabular}{lcc} 
& Infected persons (cases) & Healthy co-diners \\
\hline Number & 17 & 7 \\
Median age (range), years & $48(29-75)$ & $45(29-47)$ \\
$\leq 39$ years & $5(29 \%)$ & $1(14 \%)$ \\
$40-59$ years & $6(35 \%)$ & $5(71 \%)$ \\
$\geq 60$ years & $6(35 \%)$ & 0 \\
Unknown & 0 & $1(14 \%)$ \\
Sex: men & $12(71 \%)$ & $4(57 \%)$ \\
\end{tabular}

listed in Box 3. The highest attack rates were in those who consumed pork liver pâté, pork chorizo or roast pork. All 15 patients who provided a food history reported consuming pork liver pâté, compared with four of the seven uninfected co-diners $(P<0.05)$.

Locally acquired cases not linked to restaurant $X$. During interviews, the seven infected persons not linked to restaurant $X$ reported eating a number of pork products during their incubation periods, including supermarket ham, prosciutto, pork liver, homemade pork liver sausage, pork chops and pork belly.

Retrospective serological surveys. Of 136 serosurvey samples (31 in survey 1, 105 in survey 2), nine (6.6\%) were IgG-positive, four $(2.9 \%)$ were IgM-positive, and four $(2.9 \%)$ were both IgM- and IgG-positive for HEV. Of the eight people who were IgMpositive, HEV RNA was detected in four; sequencing confirmed infection with genotype 3 . Two of these four people reported eating at restaurant $X$ but not overseas travel, one reported travel to an HEV-endemic country, and one could not be contacted.

\section{Laboratory investigation}

HEV RNA was detected in samples from ten of the 17 restaurant $X$ cases; of the others, five with mild or no symptoms were PCR-negative, one was PCR-negative but showed seroconversion, and a sample was unavailable in one case. Sequencing of the ORF2 region was successful for all ten samples, and the HEV isolate was classified as genotype 3 . There was at least $99 \%$ between-sample sequence homology in the targeted portion of ORF2 among restaurant $X$ isolates.

HEV RNA was also detected in six of the seven locally acquired infections not linked to restaurant $X$ (the specimen supplied by one person was insufficient for testing): five were genotype 3 , and one sample was insufficient for genotyping. The viral sequence of these samples was about $90 \%$ homologous with samples from the restaurant-linked cases. 


\begin{tabular}{|c|c|c|c|c|c|c|c|c|}
\hline & \multicolumn{3}{|c|}{ Number of people who ate the item } & \multicolumn{3}{|c|}{$\begin{array}{c}\text { Number of people who did not } \\
\text { eat the item }\end{array}$} & \multirow[b]{2}{*}{$\begin{array}{l}\text { Risk ratio } \\
(95 \% \mathrm{Cl})\end{array}$} & \multirow[b]{2}{*}{$P$} \\
\hline & $\begin{array}{l}\text { Infected persons } \\
\text { (cases) }\end{array}$ & $\begin{array}{l}\text { Healthy } \\
\text { co-diners }\end{array}$ & $\begin{array}{l}\text { Attack } \\
\text { rate }(\%)\end{array}$ & $\begin{array}{l}\text { Infected persons } \\
\text { (cases) }\end{array}$ & $\begin{array}{l}\text { Healthy } \\
\text { co-diners }\end{array}$ & $\begin{array}{l}\text { Attack } \\
\text { rate }(\%)\end{array}$ & & \\
\hline Brussel sprouts & 5 & 3 & $63 \%$ & 8 & 4 & $67 \%$ & $1(0.5-1.8)$ & 1.00 \\
\hline Calamari & 3 & 2 & $60 \%$ & 10 & 5 & $67 \%$ & $1(0.5-2.0)$ & 1.00 \\
\hline Eggplant & 7 & 5 & $58 \%$ & 6 & 2 & $75 \%$ & $0.8(0.5-1.5)$ & 0.66 \\
\hline Pork chorizo & 7 & 2 & $78 \%$ & 6 & 5 & $55 \%$ & $1.5(0.8-2.7)$ & 0.36 \\
\hline Pork pâté & 15 & 4 & $79 \%$ & 0 & 3 & 0 & Undefined & 0.02 \\
\hline Roast pork & 9 & 4 & $69 \%$ & 4 & 3 & $57 \%$ & $1.2(0.6-2.6)$ & 0.64 \\
\hline
\end{tabular}

* Food histories were available for 15 of the 17 infected persons ( 13 were complete and two were incomplete) and for all seven well co-diners.

\section{Environmental investigation}

Investigation of restaurant $X$. Restaurant $X$ was found to be well managed; no breaches in food safety or handling were identified. Staff were trained in handwashing and general food safety, including understanding cross-contamination and temperature control. During the observed cooking process, the internal temperature of the pork livers reached $51^{\circ} \mathrm{C}$ at 3 minutes, and between $82^{\circ} \mathrm{C}$ and $97^{\circ} \mathrm{C}$ at 4 minutes.

The livers used for pâté preparation were traced to a single farm. The pork shoulder, jowl and chorizo products were all sourced from different suppliers to the pork livers. HEV was not detected in any of the food samples obtained from the restaurant.

Investigation of pork products of locally acquired cases not linked to restaurant $X$. Pork products eaten by the seven infected persons not linked to restaurant $X$ were bought from four different butchers and three different supermarkets. Pork livers from two of these butcheries could be traced back to two abattoirs supplied by several farms; further tracing was not undertaken. Pork liver sausages still held by one patient were found to contain very low levels of HEV RNA; the levels were too low for sequencing.

Public health interventions. NSW Health convened an expert panel involving public health, clinical, laboratory, agricultural and industry experts to assess the risks and to guide the investigation. On 15 May 2014, restaurant $X$ was informed about its possible link with a number of cases of HEV infection. The importance of thorough cooking of pork products, including of pork liver pâté, was stressed, and the restaurant voluntarily removed this item from its menu. No further cases of $\mathrm{HEV}$ infection were linked to restaurant $X$.

As part of case finding, NSW Health issued an alert to gastroenterologists and public and private pathology laboratories in May 2014. The information garnered was then used to inform general practitioners in an alert, issued in September 2014, which requested that they consider $\mathrm{HEV}$ infection in people with a compatible illness, regardless of overseas travel. A joint media release with the New South Wales Food Authority, also issued in September 2014, urged the public to cook pork products thoroughly and, in particular, to cook pork livers to $75^{\circ} \mathrm{C}$ at the thickest part for 2 minutes. ${ }^{19}$

\section{Discussion}

This is the first reported Australian outbreak of locally acquired HEV infection and one of the largest linked with a restaurant reported anywhere. Seventeen cases were linked to consuming pork liver pâté at restaurant $X$ during a 9-month period, and seven cases were linked to eating pork products bought from four butchers and three supermarkets with at least two different suppliers.

Retrospective serological testing identified a further eight previously undiagnosed cases of HEV infection (anti-HEV IgM). In two of these cases, HEV RNA was detected in people who reported no overseas travel but who had dined at restaurant $X$ during their incubation periods. A further six cases were notified after the restaurant outbreak, probably as a result of increased vigilance and testing by clinicians. Data from a large public health laboratory confirmed this, with more than triple the number of HEV tests requested and carried out from July to December 2014 (after the laboratory began testing for HEV in people without a travel history) than during the same period in 2013 (unpublished data).

Active case finding among co-diners of restaurant cases detected locally acquired HEV infections that were either asymptomatic or mildly symptomatic, suggesting underrecognition and under-diagnosis of infection. A recent HEV serosurvey of blood donors by the Australian Blood Service identified past $\mathrm{HEV}$ infection in 14 of 194 blood donors without a history of overseas travel $(7 \%){ }^{20}$ A case report in the Northern Territory $^{21}$ and a study in Victoria ${ }^{22}$ each described single cases of HEV infection in which overseas travel was not implicated and no other risk factors were identified. 
Common source outbreaks of HEV infection in high-income countries are rare. However, our investigation concurs with previous French, ${ }^{5}$ English $^{10}$ and Japanese ${ }^{11}$ studies that have linked HEV infection with consumption of undercooked pork products. In these countries, locally acquired HEV infections predominate, and in 2013 accounted for $99 \%$ of all cases in France ${ }^{23}$ and almost $70 \%$ of cases in the UK. ${ }^{24}$

$\mathrm{HEV}$ is inactivated by heating to $71^{\circ} \mathrm{C}^{19}$ Review of pork liver pâté preparation at restaurant $X$ found that it was adequately cooked at the time of inspection, and testing available pork samples did not detect HEV RNA. It is nevertheless possible that, at the time of the restaurant infections (some weeks earlier), pork livers contaminated with HEV could have been undercooked at the thickest part before blending into pâté. This may explain the relatively low proportion of patrons infected with HEV at this popular restaurant. While we did not have access to leftover pâté samples from meals served to people infected at restaurant $X$ that could be tested for HEV RNA, it was detected in pork liver sausages retained by one of the non-restaurant $X$ patients.

Most fresh pork products in Australia are locally produced. The presence of HEV in Australian pigs was first noted in 1999 by a study that reported seropositivity rates of $17 \%$ in wild-caught pigs and more than $90 \%$ in commercial pigs by 16 weeks of age. ${ }^{25}$ To our knowledge, no further studies investigating the epidemiology of HEV in Australian pigs have been conducted. Despite the link between HEV outbreaks and pork products overseas, this discovery of HEV in Australian pigs did not translate into clinical practice, perhaps because HEV was not widely recognised as being endemic to Australian pigs, and because of a lack of awareness among Australian clinicians of the veterinary literature.

A limitation to this investigation was the time lag between some infected persons and co-diners being exposed, interviewed and tested for $\mathrm{HEV}$, particularly co-diners of symptomatic persons from restaurant $X$. A lag in interviewing some infected persons and co-diners, coupled with the long incubation period of HEV (15-64 days), may have led to a recall bias in responses to the questionnaires and providing food histories. The limited sample size made it difficult to achieve statistically significant results. However, our findings are biologically plausible, and important associations could be deduced.

This study adds to our current understanding of the potential for HEV to be a food-borne illness in developed countries. Clinicians should request $\mathrm{HEV}$ testing in patients with acute hepatitis, irrespective of travel history, particularly where no aetiology has been determined. Laboratories should test for HEV where indicated to prevent underrecognition of infection. Health departments must be aware of the possibility of underestimating the prevalence of hepatitis E when using surveillance data. Pork products, particularly pork livers, should be cooked until they reach $75^{\circ} \mathrm{C}$ at the thickest part for 2 minutes.

Increased awareness, ongoing research and collaboration between primary industries, animal and human health authorities should help detect and prevent this and other emerging infectious diseases in Australia.

Acknowledgements: We express our special thanks to staff who conducted HEV testing at the Centre for Infectious Diseases and Microbiology Laboratory Services and Royal Prince Alfred Hospital, to staff at Douglass Hanly Moir Pathology for their contribution to the retrospective serostudy and to all other laboratories that contributed data. We also thank Jennie Musto, Michael Staff, Troy Mcneill, Neil Franklin, Peter Kirkland, Deborah Finlaison, Brett Campbell and Edward Jansson for their roles in the investigations.

Competing interests: No relevant disclosures. Received 20 Aug 2015, accepted 22 Dec 2015. (c) 2016 AMPCo Pty Ltd. Produced with Elsevier B.V. All rights reserved. 
1 Kamar N, Bendall R, Legrand-Abravanel F. Hepatitis E. Lancet 2012; 379: 2477-2488.

2 Pérez-Gracia MT, Suay B, Mateos-Lindemann ML. Hepatitis E: an emerging disease. Infect Genet Evol 2014; 22: 40-59.

3 Heymann DL, editor. Control of communicable diseases manual: an official report of the American Public Health Association. 19th edition. Washington, DC: American Public Health Association, 2008; p 299.

4 Christou L, Kosmidou M. Hepatitis E virus in the Western world - a porkrelated zoonosis. Clin Microbiol Infect 2013; 19: 600-604.

5 Colson P, Borentain P, Queyriaux B, et al. Pig liver sausage as a source of hepatitis $E$ virus transmission to humans. J Infect Dis 2010; 202: 825-834.

6 Said B, ljaz S, Kafatos G, et al. Hepatitis E outbreak on cruise ship. Emerg Infect Dis 2009; 15: 1738-1744.

7 Tamada $\mathrm{Y}$, Yano $\mathrm{K}$, Yatsuhashi $\mathrm{H}$, et al. Consumption of wild boar linked to cases of hepatitis E. J Hepatol 2004; 40: 869-870.

8 Tei S, Kitajima N, Takahashi K, Mishiro S. Zoonotic transmission of hepatitis $E$ virus from deer to human beings. Lancet 2003; 362: 371-373.

9 Wilhelm B, Rajić A, Greig J, et al. A systematic review/meta-analysis of primary research investigating swine, pork or pork products as a source of zoonotic hepatitis E virus. Epidemiol Infect 2011; 139: 1127-1144.

10 Said B, ljaz S, Chand M, et al. Hepatitis $E$ virus in England and Wales: indigenous infection is associated with the consumption of processed pork products. Epidemiol Infect 2014; 142: 1467-1475.
11 Miyashita K, Kang JH, Saga A, et al. Three cases of acute or fulminant hepatitis $E$ caused by ingestion of pork meat and entrails in Hokkaido, Japan: zoonotic food-borne transmission of hepatitis $\mathrm{E}$ virus and public health concerns. Hepatol Res 2012; 42: 870-878.

12 Wenzel JJ, Preiß J, Schemmerer M, et al. Detection of hepatitis E virus (HEV) from porcine livers in Southeastern Germany and high sequence homology to human HEV isolates. J Clin Virol 2011; 52: 50-54.

13 Bouwknegt M, Engel B, Herremans M, et al. Bayesian estimation of hepatitis E virus seroprevalence for populations with different exposure levels to swine in the Netherlands. Epidemiol Infect 2008; 136: 567-576.

14 Drobeniuc J, Favorov MO, Shapiro CN, et al. Hepatitis E virus antibody prevalence among persons who work with swine. J Infect Dis 2001; 184: 1594-1597.

15 Meng $X$, Wiseman $B$, Elvinger $F$, et al. Prevalence of antibodies to hepatitis $E$ virus in veterinarians working with swine and in normal blood donors in the United States and other countries. J Clin Microbiol 2002; 40: 117-122.

16 Ward K, Franklin N, Furlong C, et al. NSW 2013 OzfoodNet annual report. http://www.health.nsw.gov.au/ Infectious/foodborne/Documents/ NSW-ofn-annual-report-2013.pdf (accessed Feb 2015).

17 Gu X, Davis RJ, Walsh SJ, et al. Longitudinal study of the detection of Bluetongue virus in bull semen and comparison of real-time polymerase chain reaction assays. $J$ Vet Diagn Invest 2014; 26: 18-26.

18 Jothikumar N, Cromeans TL, Robertson $\mathrm{BH}$, et al. A broadly reactive one-step real-time RT-PCR assay for rapid and sensitive detection of hepatitis E virus. J Virol Methods 2006; 131: 65-71.

19 Feagins A, Opriessnig T, Guenette D, et al. Inactivation of infectious hepatitis $\mathrm{E}$ virus present in commercial pig livers sold in local grocery stores in the United States. Int J Food Microbiol 2008; 123: 32-37.

20 Shrestha AC, Seed CR, Flower RL, et al. Hepatitis $E$ virus and implications for blood supply safety, Australia. Emerg Infect Dis 2014; 20: 1940-1942.

21 Heath T, Burrow J, Currie BJ, et al. Locally acquired hepatitis $\mathrm{E}$ in the Northern Territory of Australia. Med J Aust 1995; 162: 318-319.

22 Cowie BC, Adamopoulos J, Carter K, Kelly H. Hepatitis E infections, Victoria, Australia. Emerg Infect Dis 2005; 11 : 482-484.

23 French Institute for Public Health Surveillance. Hepatitis E Epidemiological data [French]. 2014. http://www.invs.sante.fr/Dossiersthematiques/Maladies-infectieuses/ Zoonoses/Hepatite-E/Donneesepidemiologiques (accessed June 2015).

24 Public Health England. Public health operational guidelines for hepatitis $\mathrm{E}$. Health protection response to reports of hepatitis $E$ infection. Version 2.0. London: PHE, 2015. https://www.gov.uk/government/ uploads/system/uploads/attachment data/file/396909/PH Operational Guidelines for HepE 051214 Standard template_CT.pdf (accessed Jan 2016).

25 Chandler JD, Riddell MA, Li F, et al. Serological evidence for swine hepatitis $E$ virus infection in Australian pig herds. Vet Microbiol 1999; 68: 95-105. 